\title{
The unique evolutionary pattern of the Hydroxyproline-rich glycoproteins superfamily in Chinese white pear (Pyrus bretschneideri)
}

Huijun Jiao, Xing Liu, Shuguang Sun, Peng Wang, Xin Qiao, Jiaming Li, Chao Tang, Juyou Wu, Shaoling Zhang and Shutian Tao*

\begin{abstract}
Background: The hydroxyproline-rich glycoprotein (HRGP) superfamily, comprising three families (arabinogalactanproteins, AGPs; extensins, EXTs; proline-rich proteins, PRPs), is a class of proline-rich proteins that exhibit high diversity and are involved in many aspects of plant biology.

Results: In this study, 838 HRGPs were identified from Chinese white pear (Pyrus bretschneideri) by searching for biased amino acid composition and conserved motifs. 405 HRGPs were derived from whole genome duplication (WGD) events which is suggested to be the major force of driving HRGPs expansion and the recent WGD event shared by apple and pear generated most duplicated HRGPs in pear. This duplication event drived the structural variation of the HRGPs encoding hydroxyproline (Hyp)-rich motifs. The rate of HRGPs evolution mainly impacted the Hyp-rich motifs even in chimeric HRGPs. During the evolution of 53 PRPs that are also typified by 7-deoxyloganetin glucosyltransferase-like genes, the duplication from PRP to non-PRP was indirectly modified by positive selection. These results suggested that the rate of HRGP evolution mainly influenced the Hyp-rich motifs even in chimeric HRGPS. The expression divergence of HRGPS was higher than that of other commonly duplicated genes. In pear pistil, 601 HRGPs exhibited expression, while in pear pollen, 285 HRGPs were expressed. The qPCR results revealed that Pbr036330.1 and Pbr010506.1 showed different expression profile in self-incompatibility of pear pistil.
\end{abstract}

Conclusions: The researches indicated that WGD events was the main duplication type during the evolution of HRGPs, and the highly variable Hyp-motifs might be accountable for the expansion, evolution and expression divergence of HRGPs and that this divergence may be responsible for the gain of new functions in plants.

Keywords: Evolutionary pattern, Expression divergence, Gene duplication, HRGPs, Hyp-rich motif, Positive selection

\section{Background}

Hydroxyproline-rich glycoproteins (HRGPs) is a superfamily that contains three subfamilies: highly glycosylated arabinogalactan-proteins (AGPs), moderately glycosylated extensins (EXTs) and lightly glycosylated proline-rich proteins (PRPs) [1,2], all of which are usually post-translationally modified by prolyl 4hydroxylase, generating hydroxyproline (Нyp) residues.

\footnotetext{
*Correspondence: taost@njau.edu.cn

Centre of Pear Engineering Technology Research, State Key Laboratory of Crop Genetics and Germplasm Enhancement, Nanjing Agricultural University, Nanjing 210095, China
}

HRGPs are expressed widely in the plant kingdom, from owering plants, indicating that they play fundaleaves and reproductive tissues. Pear fertility is a key concern due to the fact that pear is a major commercial crop, and the role of HRGPs in the reproductive development of the pear is of particular interest.

The arabinogalactan chains of AGPs are usually linked to galactose by Hydroxyproline-Galactose (Hyp-Gal) 
linkages. Recent studies demonstrated that AGPs play vital role in many tissues, such as leaves, roots and floral tissues [4]. AGPs often lie in the cell surface and are implicated in vegetative and reproduction growth $[2,5]$. T. Schindler reported that AGPs are involved in plant development, such as the xylem differentiation [6]. AGP31 (At1g28290) participates in cell wall formation by interacting with various cell wall components [7]. AGP19 (At1g68725) is involved in cell division and expansion [8]. AGPs expressed in the transmitting tissue of the style also assist pollen adhesion on the stigma and direct pollen tube growth along the transmitting tissue $[9,10]$. AGPNa3 protein (RT35 protein) is localized in the stigma, specifically in the pistil of Nicotiana alata (tobacco) [11]. $120 \mathrm{kDa}$ glycoprotein (120 K) expressed in the style plays a role in S-specific pollen rejection in $N$. alata and is taken into the pollen tube [12]. The AGPenriched, stylar-transmitting canal exudate of Lilium longittorum has been shown to serve as an adhesive matrix that enhances pollen tube growth in vitro [13].

EXTs contains several repetitive $\mathrm{SP}_{\mathrm{n}}$ motifs in their Hyp-rich motifs. Most EXTs belonging to insoluble cell wall proteins are expressed in root, pollen/stamen or siliques [14]. EXTs are involved in nearly all aspects of plant growth and development, including pollen recognition, fertilization [15], defence responses [16] and cell division, differentiation and elongation [17-19]. The repetitive $\mathrm{SP}_{\mathrm{n}}$ motifs of Pex protein may participate in species-, family- or tissue-specific recognition in Solanum lycopersicum (tomato) [20]. Pex1 expressed in Zea mays pollen interacts with a specific partner and triggers the downstream reaction [21]. AtLRX1 is directly involved in the process of cell wall formation in Arabidopsis thaliana. The $\mathrm{SP}_{\mathrm{n}}$ motifs in LRX proteins with variable length and number are involved in regulating signalling processes in cell walls [22].

Proteins rich in prolines or Hyps cannot be classified into EXTs or AGPs, and they are defined as PRPs [23]. PRPs are mostly expressed in endosperm, shoot apex and petiole, while a few are expressed in root [14] or are involved in reproduction. PRPs in Glycine max (SbPRP1, SbPRP2, SbPRP3) are expressed in various kinds of cells and are distinct from cell wall proteins. ENOD2 isolated from Pisum sativum is important for cell morphology, especially in the nodule parenchyma [24]. HyPRP in maize is highly expressed in zygotic embryo and ovary prior to pollination and is thought to contribute to the stability and defence of the developing embryo [25].

Gametophytic self-incompatibility (SI) is a widespread mechanism that prevents inbreeding and promotes outcrossing in flowering plants [26]. SI of plants belonging to the genera Rosaceae, Solanaceae and Plantaginaceae is determined by S-RNase [27]. Pollen tube growth in the extracellular matrix (ECM) triggers a series of interactions between pollen and pistil. For instance, AGP components of the stylar ECM directly interact with pollen tubes in tobacco [12]. AGPNa3 protein (RT35 protein) located in the stigma may have a specific role in pistil [11]. Transmitting tissue-specific glycoprotein (NaTTS) was reported to be involved in attracting pollen tubes in vitro and stimulating pollen tubes both in vivo and in vitro. NaTTS protein levels are highly reduced in transgenic tobacco plants, which leads to a reduction of pollen tube growth rate $[28,29]$. The class III pistil-specific extensin-like (PELPIII) proteins were reported to incorporate into both compatible and incompatible pollen tube $[12,30] .120 \mathrm{~K}$ is taken into pollen tube and is associated with pollen tube vacuoles [29, 31]. $120 \mathrm{~K}$, PELPIII and TTS are able to bind S-RNase, while only $120 \mathrm{~K}$ is necessary for SI [12, 28, 29]. However, the role of most HRGPs in the SI of pear remains unclear.

Herein, we studied pear HRGPs by investigating phylogenetic relationships, evolutionary patterns and expression patterns in reproductive tissues. In contrast to the bioinformatics analysis of HRGPs in Arabidopsis and Populus trichocarpa (black cottonwood) [32, 33], our data suggested the evolution patterns and expression divergence influenced by Hyp-rich motifs and elucidated the importance of Hyp-rich motifs in HRGPS evolution. Finally, the expression patterns of HRGPS in reproductive tissues were analysed to uncover their functional roles in SI.

\section{Results}

\section{Identification and classification of HRGPs in pear}

In order to collect all HRGPs in pear, multiple search methods based on the features of each family were utilized. Based on biased amino acid composition and conserved motifs, a total of 838 HRGPS were identified, including 522 AGPs, 201 PRPs and 115 EXTs (Additional file 1: Table S1). However, 56 HRGPs were identified as repetitive sequences during screening. Based on the characteristics of Hyp-rich motifs, HRGPs were classified into three families: AGPs, EXTs and PRPs.

The percentage, sort order and the amount of proline $(\mathrm{P})$, alanine $(\mathrm{A})$, serine $(\mathrm{S})$ and threonine $(\mathrm{T})$ were regarded as criteria for identifying AGPs, and 522 sequences were collected (Table 1). By a detailed identification of sequence characters, the AGP family was divided into five subfamilies: AG (arabinogalactan) peptides, lysine-rich AGPs, FLAs (fasciclin-like AGPs), EXT-AGPs and classical AGPs. 126 AG peptide sequences were identified by searching for biased amino acid (aa) compositions with at least 10\% PAST and protein length less than or equal to 90 aa. 183 Lys-Rich AGPs were identified by searching for biased aa compositions with at least $10 \%$ PAST and lysine-rich motifs. 7 FLAs proteins containing fasciclin $\mathrm{H} 1$ motifs with more 
Table 1 Identification and classification of HRGPs in pear genome

\begin{tabular}{|c|c|c|c|c|c|}
\hline Family & Subfamily & Searching Strategies & HRGPs with SP & HRGPs with GPI & Total \\
\hline \multirow[t]{3}{*}{ EXT } & SP3 & $>=2 \mathrm{SPPP}$ & 19 & 3 & 56 \\
\hline & SP4 & $>=2$ SPPPP & 16 & 2 & 33 \\
\hline & SP5 & $>=2$ SPPPPP & 16 & 0 & 26 \\
\hline \multirow[t]{5}{*}{ AGP } & EXT-AGPS & $>10 \%$ PAST\& SP motif & 33 & 4 & 54 \\
\hline & AG-peptides & $>10 \%$ PAST \& AA < 90 & 15 & 5 & 126 \\
\hline & lysine-AGPs & $>$ 10\%PAST\& Lysine rich Domain & 33 & 10 & 183 \\
\hline & FLAs & > 10\%PAST\&Fasciclin Domain & 5 & 3 & 7 \\
\hline & Classical AGPs & $>30 \%$ PAST & 44 & 24 & 152 \\
\hline PRP & PRP & PPV.[KC] & 17 & 0 & 201 \\
\hline
\end{tabular}

Note: SP refers to signal peptide; GPI refers to glycosylphosphatidylinositol

than 10\% PAST were identified. 54 EXT-AGPs were identified by searching for $[\mathrm{SP}]_{3,4,5}$ motifs with at least 10\% PAST content. 152 Classical AGPs were identified by searching for biased aa compositions with at least $30 \%$ PAST and no swapped domains. Comparing the size of the subfamily, members of Lys-Rich AGPs > Classical AGPs > AG peptides > EXT-AGPs, and only 1\% of AGPs were FLAs. The signal peptides (SPs) cleavage sites and glycosylphosphatidylinositol (GPI) anchor sites were also predicted. $25 \%$ and $9 \%$ of AGPs members were annotated with SPs and GPI anchors, respectively. The prototypical structure of each subfamily member is displayed in Additional file 2: Fig. S1.

115 EXTs genes were identified by screening pear protein database with more than $2 \mathrm{SP}_{3}, \mathrm{SP}_{4}$ and/or $\mathrm{SP}_{5}$ motifs. EXT family members were divided into three subfamilies based on the number of Hyp-rich repeats in the motifs of $\mathrm{SP}_{3}$-EXT, $\mathrm{SP}_{4}$-EXT and $\mathrm{SP}_{5}$-EXT. The $\mathrm{SP}_{3}$ EXT subfamily consisted of 56 members, of which 19 have SPs cleavage sites and 3 have GPI anchor sites. The $\mathrm{SP}_{4}$ EXT subfamily consisted of 33 members and 16 have SPs cleavage sites and 2 have GPI anchor sites among them. The $\mathrm{SP}_{5}$-EXT subfamily consisted of 26 members and 16 of them have SPs cleavage sites and none has a GPI anchor site (Table 1). The typical structure of the EXT family is displayed in Additional file 2: Fig. S1.

201 PRPs were identified by screening [KKPCPP] and $[\operatorname{PPVX}(\mathrm{K} / \mathrm{T})]$ motifs against the pear protein database. Only 17 PRPs members were predicted to have SPs, while none was found to have a GPI anchor site (Table 1 ). The typical structure of PRP family is displayed in Additional file 2: Fig. S1. The classification results suggested that our searching strategy is accurate.

\section{Phylogenetic analysis of the HRGP superfamily}

Considering that the sequences structure of HRGPs from different families vary greatly, the phylogenetic tree of total superfamily members can not reflect the influence of biased aa composition on phylogenetic relationships. To survey phylogenetic relationships, the phylogenetic trees of each family were constructed using RAxML. The phylogenetic tree of AGP family was constructed based on the percentage of PAST and absolute number of PAST sequences in each member. Members of each subfamily did not cluster together, suggesting that the phylogenetic relationship was not affected by biased amino acid composition. However, AGPs with similar amount of PAST always clustered together, while AGPs with contrasting percentages of PAST showed a balanced clustering (Fig. 1). The phylogenetic tree of EXT family was constructed and combined with numbers of $\mathrm{SP}_{\mathrm{n}}$ motifs (Additional file 3: Fig. S2). Members of each EXT subfamily spread evenly between the clades, suggesting that the phylogenetic relationship was not in accordance with the type of $\mathrm{SP}_{\mathrm{n}}$ motifs. However, EXTs with relatively more $\mathrm{SP}_{\mathrm{n}}$ motifs tended to cluster together.

The phylogenetic tree of PRP family was also constructed using amino acid sequences (Additional file 4: Fig. S3). Most PRPs with PPV $[\mathrm{X}] \mathrm{C}$ motifs clustered together and were mainly distributed in two branches. PRPs with PPV $[\mathrm{X}] \mathrm{K}$ clustered in a very scattered manner, suggesting that PRPs with this Hyp-rich motif may have more extensive functionality. The topological structures of three phylogenetic trees suggested that the evolutionary pattern of HRGPs is complexity.

\section{Expansion patterns of the HRGP superfamily}

To further ascertain the underlying discordance mechanism between phylogenetic relationship and biased aa composition, we determined the expansion patterns of HRGPS. It has been demonstrated that whole genome duplication (WGD), segmental duplication and singlegene duplication are the major gene duplication modes [34]. Five modes of gene duplication in the HRGP superfamily were identified using MCSanX, including WGD, segmental, tandem, dispersed and proximal duplication. The duplication types for HRGP family members were 


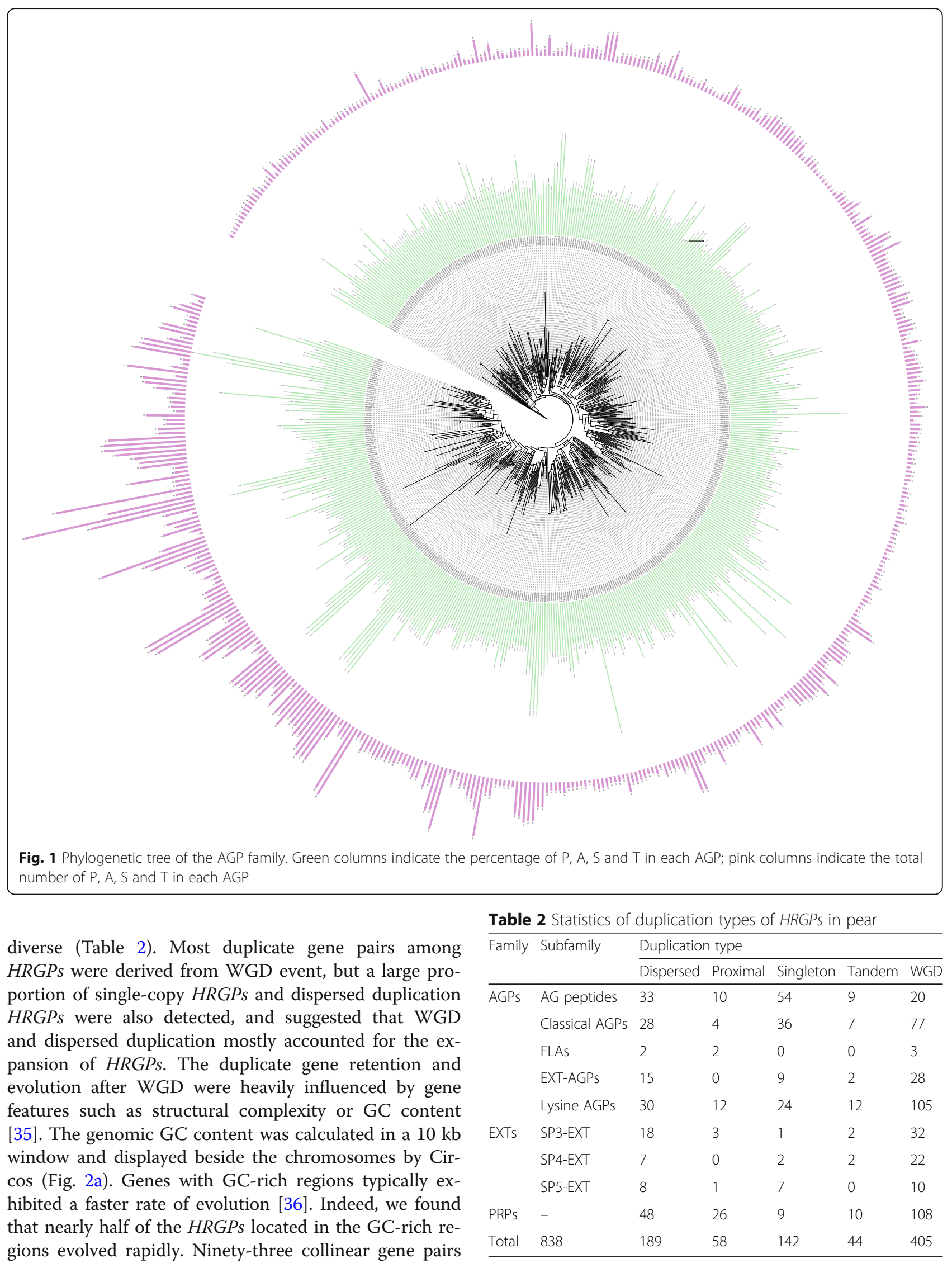




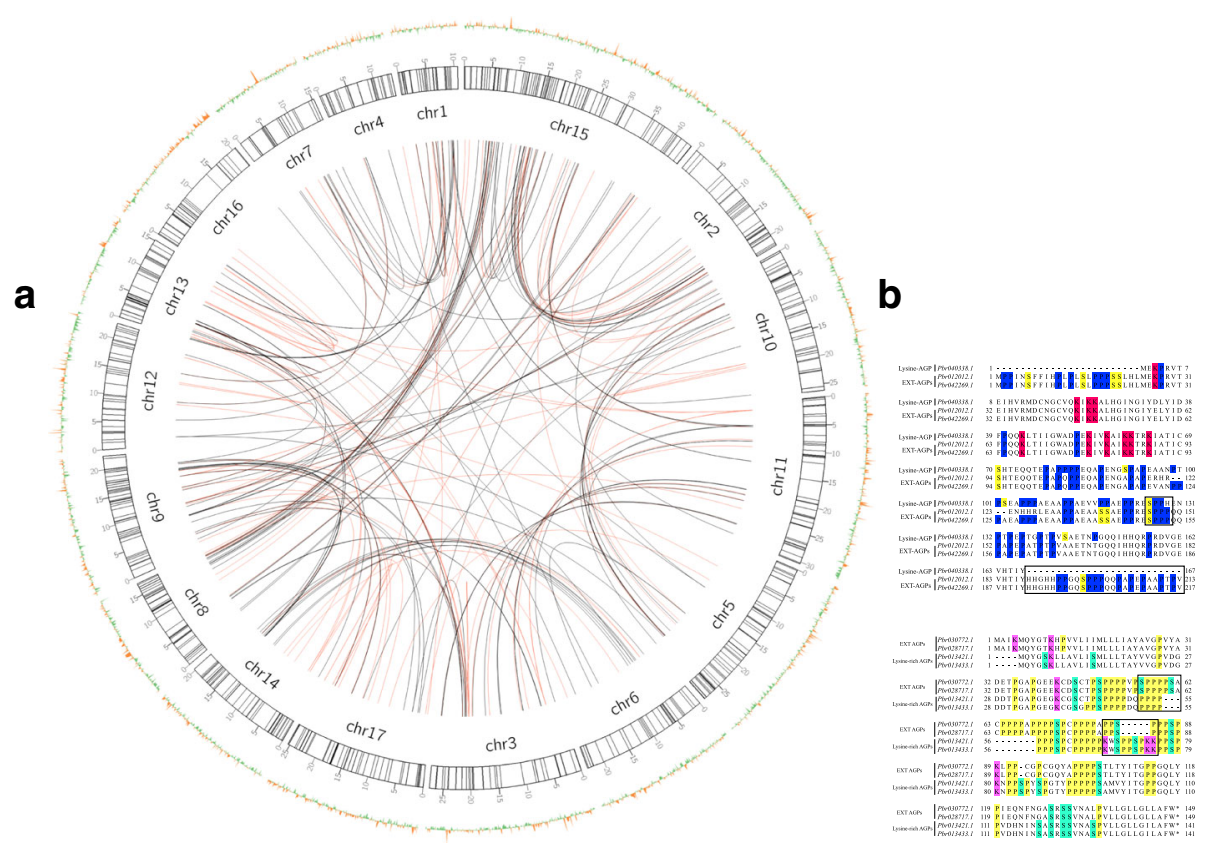

Fig. 2 Gene duplication analysis of HRGPs in pear. a. Gene pairs within HRGPS are joined by red lines; gene pairs in which only one duplicated gene is an HRGP are joined by black lines. Average GC content is indicated by coloured columns outside the chromosomes. Regions with high GC content are indicated by red columns, and low GC regions are indicated by green columns. $\mathbf{b}$. The alignments of two collinear gene pairs between EXT-AGPs and lysine-rich AGPS. The mutant amino acid sequences are indicated by black boxes

were found among all HRGPs, while no PRP gene was found to be collinear with other HRGPs. Collinear gene pairs were located in homologous chromosome regions, such as 9 and 17, 8 and 15, which descended from the recent WGD event occurred in pear [37]. This result indicated that the recent WGD event shared by pear and apple generates numerous HRGPs (Fig. 3). We observed a number of gene pairs in which the two gene copies belonged to different subfamilies of HRGPs, indicating frequent structure variations within the family. According to the sequence alignments of chimeric HRGPs, we found that Hyp-rich motifs were much more variable than common conserved domains and might be responsible for the high variation of HRGPs after gene duplication.

We then performed sequence alignment of collinear gene pairs in which the two gene copies belonged to different subfamilies in order to analyze the structural variation of HRGPs such as Pbr028717.1 (EXT-AGP), Pbr013421.1 (lysine-rich AGP), Pbr030772.1 (EXT-AGP) and Pbr013433.1 (lysine-rich AGP). The results demonstrated that $\mathrm{P}_{52}$ in the two lysine-rich AGPs had mutated to $\mathrm{S}_{56}$, putting Pbr030772.1 and Pbr028717.1 into the EXT-AGPs subfamily. In addition, the loss of $K_{67}, K_{74}$ and $K_{75}$ resulted in the absence of lysine-rich motifs in Pbr030772.1 and Pbr028717.1 (Fig. 2b). Additionally, Pbr042269.1 (EXTAGP) were collinear with Pbr012012.1 (EXT-AGP) and Pbr040388.1 (lysine-rich AGP). Each of them contained a typical lysine motif of KIVKAIKKTRK, while both Pbr042269.1 and Pbr012012.1 contained two extra SPPP motifs. Compared with the two EXT-AGPs, $\mathrm{P}_{129}$ in Pbr040338.1 mutated into $\mathrm{H}_{129}$ and a set of amino acids marked with a black box were deleted, including an SPPP motif. These mutations changed Pbr040338.1 into a typical lysine-rich AGP (Fig. 2b). These sequence variations between duplicate pairs among HRGPs indicated that the Hyp-rich motif was particularly variable and affected AGP classification. Furthermore, the duplication between HRGPs and non-HRGPs was much more frequent than one HRGP to another HRGP, which also confirmed the high frequency of variance in Hyp-rich motifs (Fig. 2a).

\section{Evolutionary patterns of HRGPs}

Many HRGPs are chimeric proteins formed via domain swapping [38]. Considering that most conserved domains evolve under purifying selection while the variation of Hyp-rich motifs in HRGP is frequent, we explored whether Hyp-rich motifs would affect the evolutionary rate. Comparisons of evolutionary rates between chimeric HRGPs and genes with corresponding domains elucidated special duplication patterns by collinearity analysis.

The ratio of Ka (nonsynonymous substitutions per site) and Ks (synonymous substitutions per site) was widely used to estimate the evolutionary rate of coding sequences. We chose a set of chimeric HRGPs 


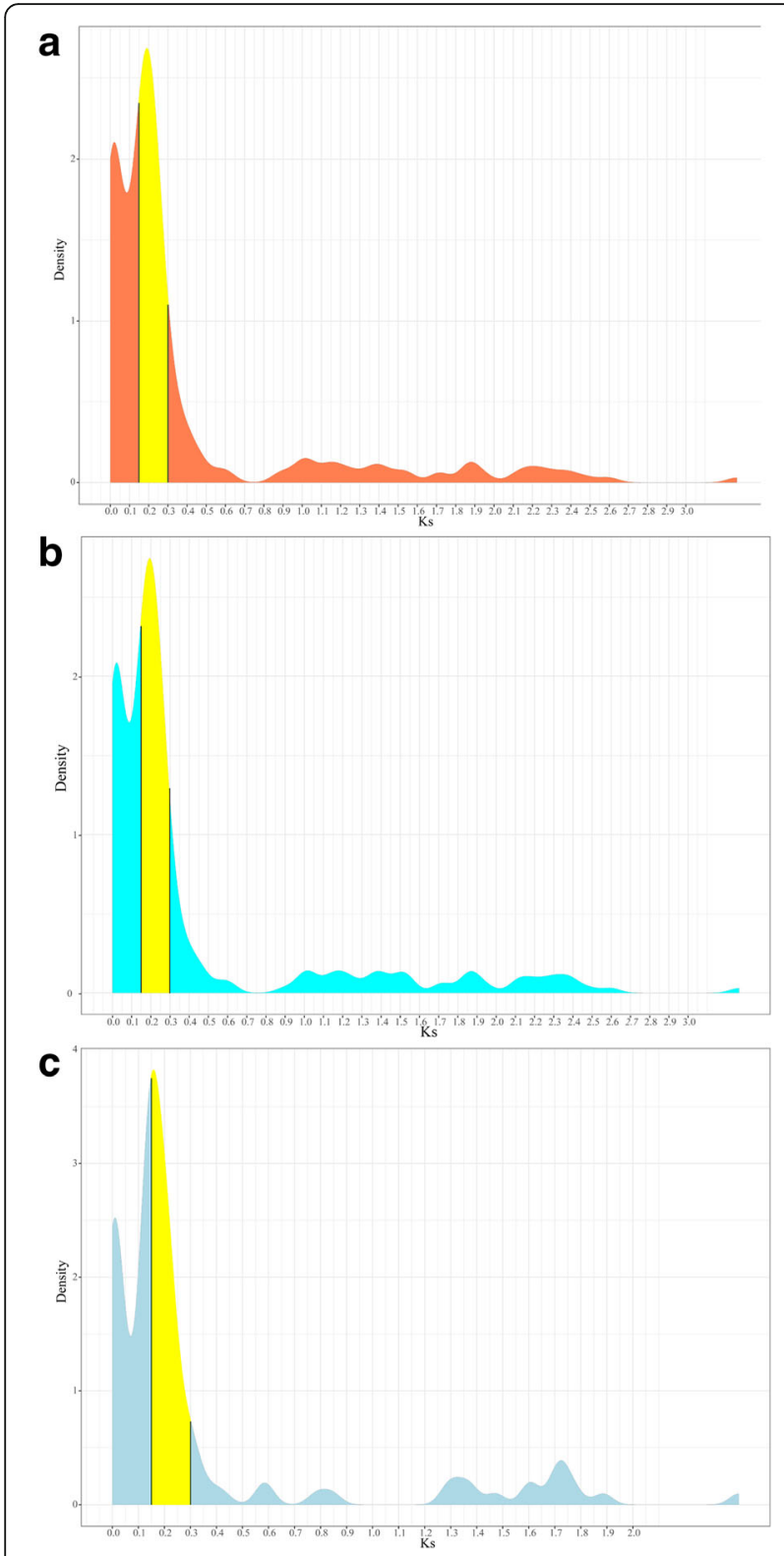

Fig. 3 The relative duplication time of HRGPS as indicated by Ks values. a-c. The relative duplication time of the AGP (A), EXT (B) and $P R P(C)$ families. The yellow region ranging from 0.15 to 0.3 indicates the recent WGD events shared by pear and apple

containing conserved domains for further analysis, such as leucine-rich repeats (LRR, PF12799.1), Pkinase_Tyr (PF07714.15), probable lipid transfer (LTP, PF14368.4) and RNA recognition motifs (RRM, PF00076.20). The selected HRGPs were used to compare the evolutionary rates between chimeric HRGPs and proteins with similar swapped domains (Fig. 4). We found that the evolutionary rates between chimeric HRGPs and genes with corresponding domains were significantly different. Specifically, for chimeric HRGPs with LRR and LTP motifs, the evolutionary rate of HRGPs was higher than that of non-HRGPs. For chimeric HRGPs with PKinase_Tyr and RRM, the evolutionary rate of HRGPs was lower. It suggested that in different chimeric HRGPs, the Hyprich motif did not simply accelerate or decelerate the evolutionary rate. We also compared the evolutionary rate among chimeric HRGPs, and no significant difference was found by T-test. It suggested that Hyp-rich motifs were likely to be more influential than conserved domains in chimeric HRGPs on the evolutionary rate.

Three phylogenetic trees were constructed via RAxML using aa sequences. Genes clustered in each branch presented a close phylogenetic relationship. Analyzing the PRP phylogenetic tree and the Hyp-rich motif features of each branch, we found that most PRPs with a $\mathrm{PPV}[\mathrm{X}] \mathrm{C}$ motif belonged to a specific gene family (Additional file 4: Fig. S3).

To explore the formation of chimeric HRGPs, we analyzed the 7-deoxyloganetin glucosyltransferase-like gene family containing both PRPs and non-PRPs as a representative set. Using PRP Pbr024572.1 as the query sequence to blast against the pear proteome, we found 266 homologous genes belonging to 7-deoxyloganetin glucosyltransferase-like gene family. Fifty-three closely related genes containing both PRPs and non-PRPs were retained. We found PRPs spread among all phylogenetic branches, indicating that an ancient gene may have contained this motif.

We used codeml from the PAML package to estimate the selection pressure for 53 genes (Fig. 5a). We found that most of the sites were under negative selection (Fig. 6a), suggesting that genes in the tree evolved conservatively; however, 7 amino acid sites under positive selection were also found. In the blue clade, most of the genes were identified as PRPs, while Pbr040614.1 mutated into a non-PRP. Among all estimated sites, one positively selected amino acid site $\mathrm{S}_{120}$ in Pbr040614.1 mutated to $\mathrm{K}_{120}$ (Fig. $6 \mathrm{~b}$ ). The mutation of this site may trigger a change of Pbr040614.1 in function and result in the structural variation of its Hyp-rich motif. However, the PRP Hyp-rich motif PPV $[\mathrm{X}] \mathrm{C}$ was under significant purifying selection, implying that the motif of $\mathrm{PPV}[\mathrm{X}] \mathrm{C}$ was conservative (Fig. 5b, c). It seemed that this Hyp-rich motif was not directly modulated by positive selective pressure, while other sites under positive selection could lead to the structural variation of Hyp-rich motif in PRPs.

The divergence time of the blue clade ranged from 0.45 to 0.52 , during which 7-deoxyloganetin glucosyltransferaselike genes of pear experienced an explosive expansion (Fig. 6c). The divergence time of Pbr040614.1 and the other PRPs ranged from 0.46 to 0.49 and was also within the time of rapid expansion. We also found that during the expansion mode of 7-deoxyloganetin glucosyltransferase-like genes, there was no WGD event which is a major force to expand most gene families. The explosive expansion might 

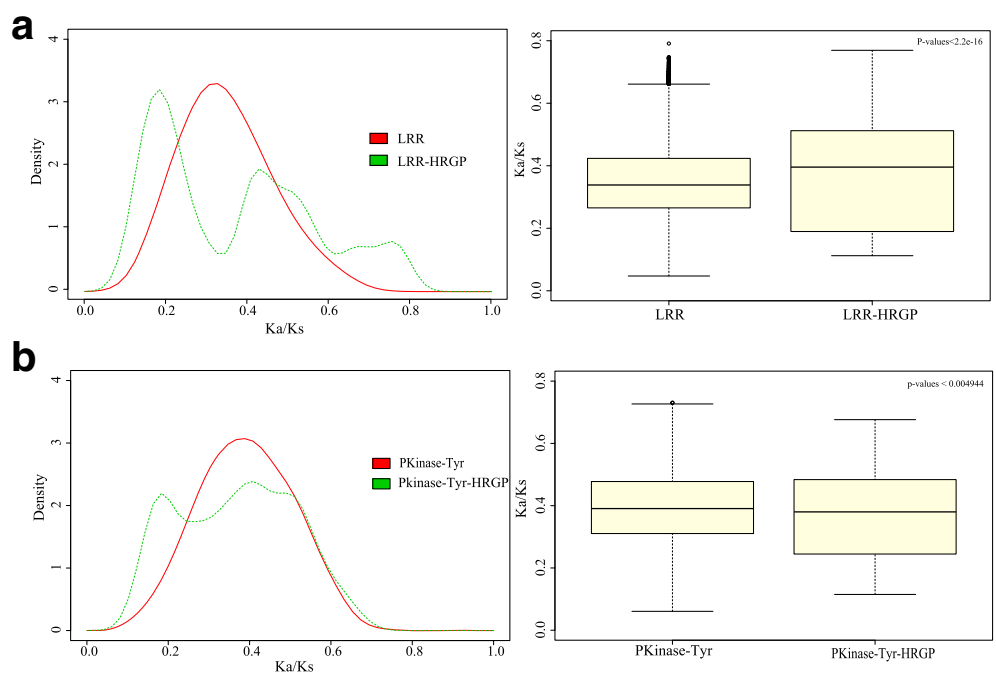

C
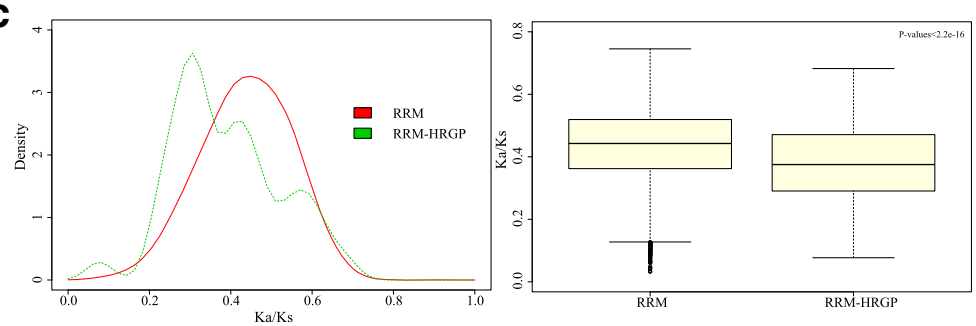

d
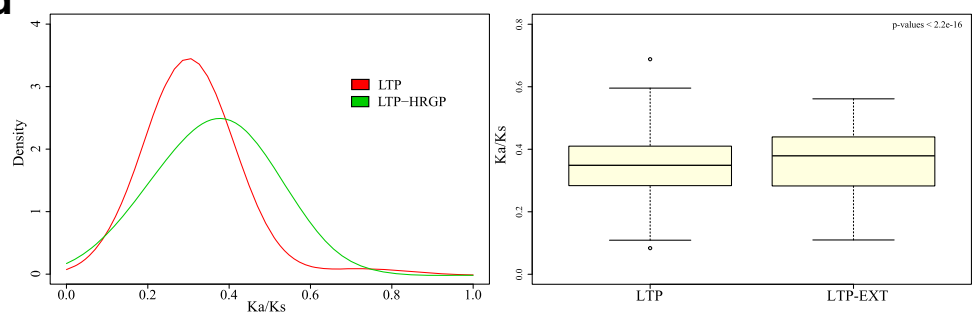

Fig. 4 Comparison of evolutionary rates between chimeric EXTs and corresponding homology genes without proline-rich motifs. a Comparison of evolutionary rates between LRR family and LRR-HRGP family. $\mathbf{b}$ Comparison of evolutionary rates between Pkinase-Tyr family and Pkinase-TyrHRGP family. c Comparison of evolutionary rates between RRM family and RRM-HRGP family. $\mathbf{d}$ Comparison of evolutionary rates between LTP family and LTP-HRGP family. Abbreviations: LRR: leucine-rich domain; Pkinase-Tyr: protein tyrosine kinase; RRM: RNA recognition motif; LTP: liquid transfer domain

have been caused by numerous single-gene duplications, and nearly half of the identified PRPs were found to duplicate in this manner.

HRGPs from Pollen Ole e I family typically participated in the highly divergent reproductive process in plants, making the duplication of this chimeric HRGP domain particularly important. The Pollen Ole e I domain HMM file was used as a query to perform the Hmmsearch against the pear genome. We found 42 sequences containing two PRPs, two AGPs and one EXT. The phylogenetic tree of the 42 genes was constructed and the selection pressure on aa residues was estimated. It was found that the Pollen Ole e I gene family was under positive selection and 23 amino acid sites were under strong selective pressure (Fig. 7b). We also found that Pbr026226.1, Pbr041309.1 and Pbr016247.1 were closely related in the clade, but the Pbr041309.1 was not an $A G P$. Sequence alignment suggested that Pbr041309.1 lacked an N-terminal PAST sequence. We also found that a positively selected site, $\mathrm{H}_{117}$, was lost in Pbr041309.1, while the other two genes retained this site (Fig. 7a). We speculated that the mutation of $\mathrm{H}_{117}$ might have triggered the loss of PAST fragment. These results suggested that positive selection is correlated with the gain and loss of HRGPS.

\section{Expression of HRGP superfamily genes in reproductive tissues of pear}

Because the evolutionary rates between chimeric HRGPs and genes with corresponding domains showed divergent patterns, we analysed expression pattern to further 


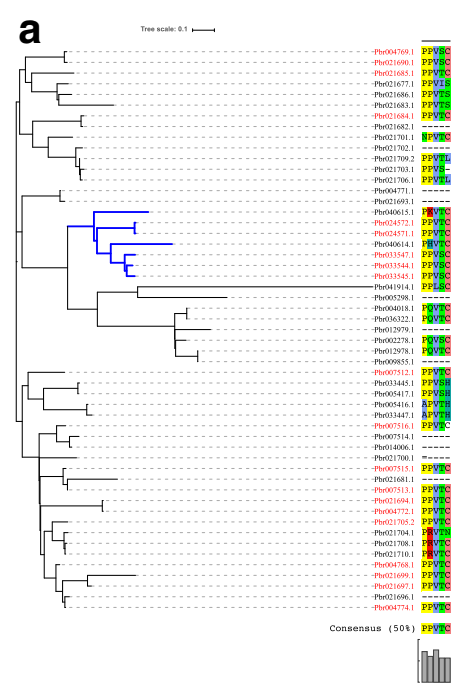

b
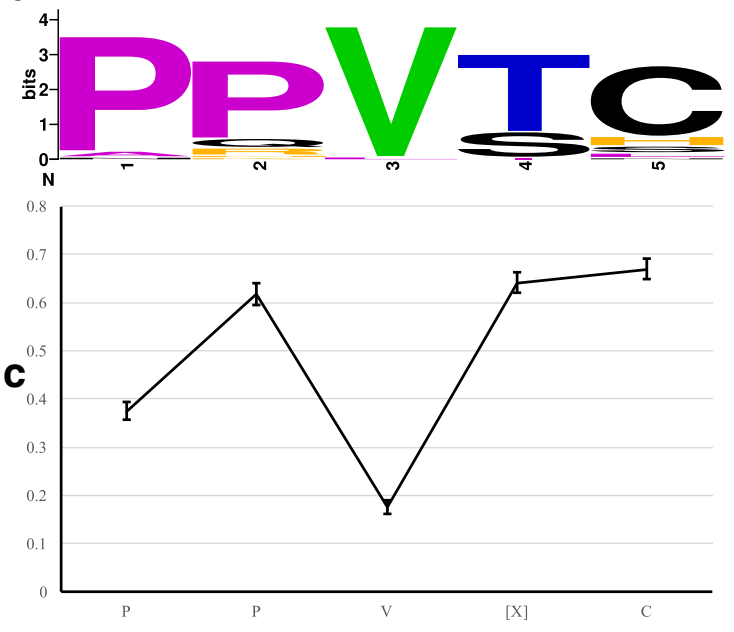

Fig. 5 The variation of Hyp-rich motifs in PRPs is affected by selective pressure. $\mathbf{a}$. The phylogenetic tree and corresponding motifs of PRPs and non-PRPs. Red taxa refer to PRPs and black taxa refer to non-PRPs. b. Amino acid compositions of 53 PRPs and non-PRPs. c. Comparison of omega among all sites within the motif

explore special chimeric HRGPs in pear. Many HRGPs were reported to play vital roles in reproductive processes, particularly in styles [39]. Therefore, we examined the expression patterns of HRGPs in pollens and styles of pear, which is a classic SI plant. To better understand the expression patterns of HRGPs, we used the RPKM values from RNA-seq in different developmental stages of the pollen tube and during the dynamic development of self- and non-self-pollinated styles (The data have not been published).
A diverse expression pattern of HRGPs in different tissues was found. We found 601 HRGPs were expressed in pollinated pistils, while only 285 were expressed in pollen. We clustered all expressed HRGPs from both transcriptome libraries (Additional file 5: Fig. S4) and found that HRGPs represented distinct expression patterns. In pollen, the highly expressed HRGP genes were less than low expressed genes, while it was opposite in pollinated style (Additional file 5: Fig. S4). This suggested that HRGP genes may play more important roles
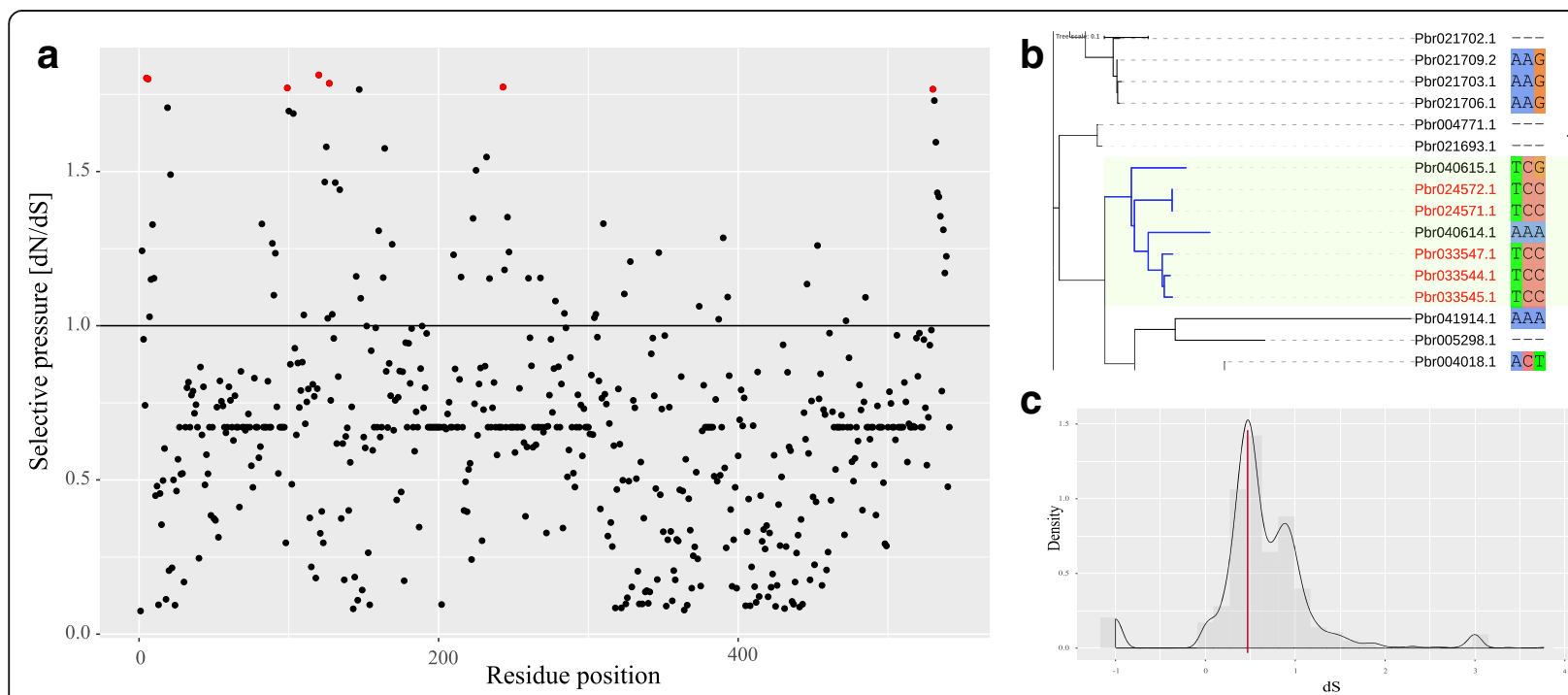

C

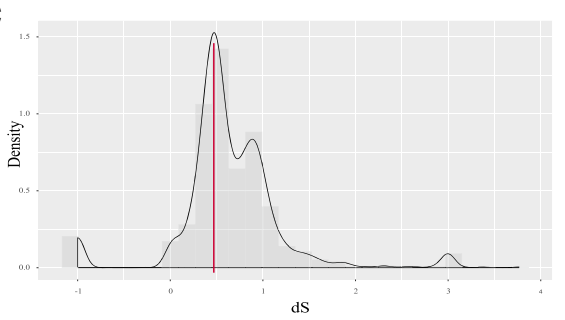

Fig. 6 The evolution pattern of PRPs in the 7-deoxyloganetin glucosyltransferase family. $\mathbf{a}$. The selective pressure acting on total amino acid sites. The significant positively selected sites are marked by red circles. b. The positively selected site $S_{120}$ mutated to $K_{120}$ among PRPs and non-PRPs. Red taxa refer to PRPs and black taxa refer to non-PRPs. $\mathbf{c}$. The divergence time of 7-deoxyloganetin glucosyltransferase family. The red line refers to 0.4871 , indicating the divergence time of PRPs and non-PRPs in the blue clade 


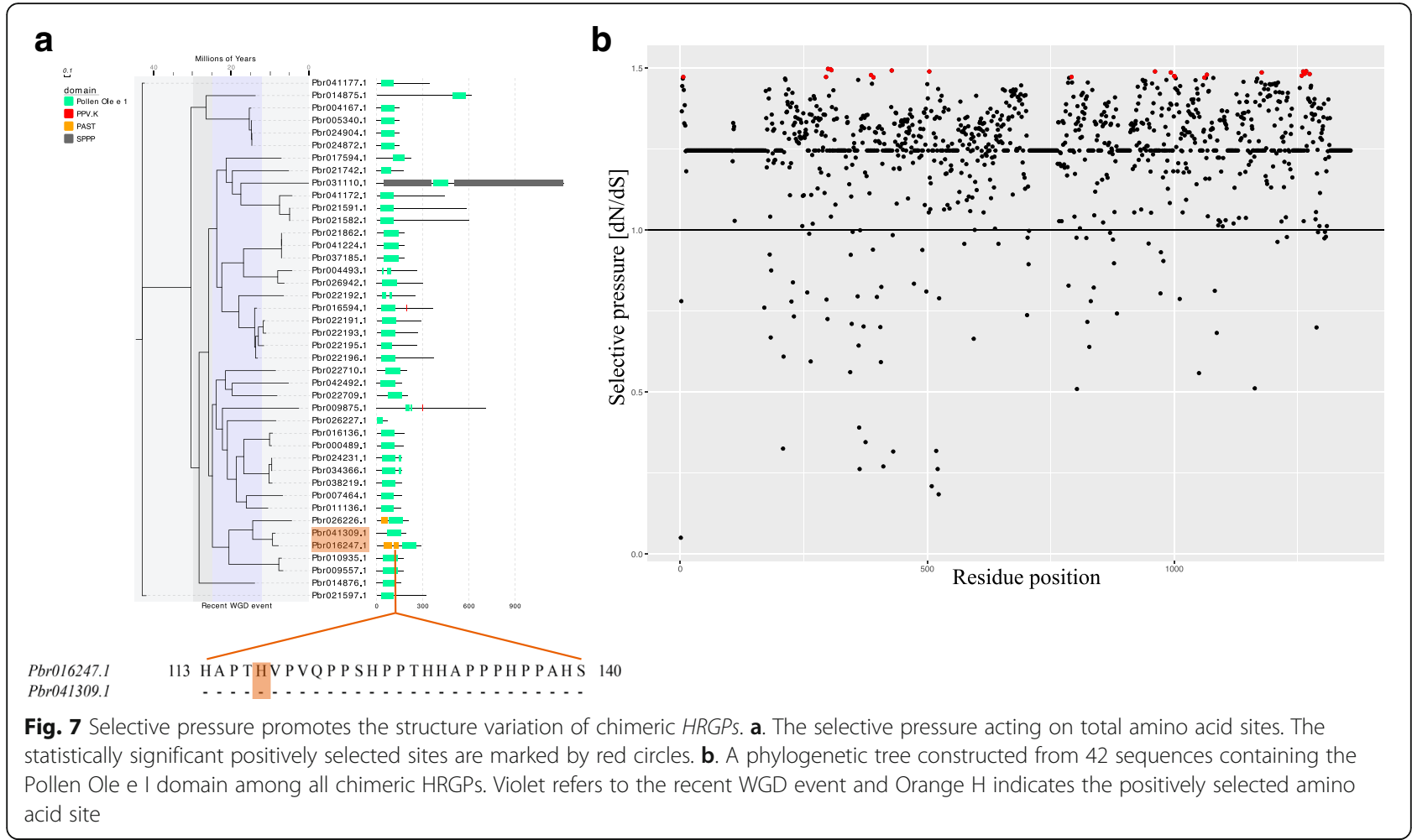

in pistils than that in pollens. These results were in accordance with previous data of TTS and $120 \mathrm{~K}$ [40]. We also found that the expression of most HRGPS was stable during different stages of pollen tubes and pistils development. We also carried out the analysis of expression pattern of HRGPs in fruit development and we found that the HRGPS showed complicated expression patterns (Additional file 6: Fig. S5).

'Jinzhui' is a self-compatible bad mutant from 'Yali' and we hypothesised that the mutant genetic features might be related to the pollen. Previous report indicated that pollination in 'Jinzhui' $\times$ 'Yali' failed, while pollination in 'Jinzhui' $\times$ 'Jinzhui' was successful [41]. However, other reports suggested that 'Jinzhui' pollen could pollinate the pistils of both 'Jinzhui' and 'Yali' [42]. Therefore, we analyzed RNA-seq of various stages of the two different pollinated pistils to reveal potential mutation mechanism between 'Yali' and 'Jinzhui'. It may be caused by the divergent expression pattern of HRGP genes during the pollination process.

To test this possibility, the pistils of 'Jinzhui' were pollinated with 'Jinzhui' or 'Yali' pollen separately and were collected at $24 \mathrm{~h}, 48 \mathrm{~h}$ and $72 \mathrm{~h}$ after pollination. After comparing the reads per kilobase per million (RPKM) of the HRGP superfamily, two HRGP genes exhibited differential expression patterns.

In 'Jinzhui $\mathrm{x}$ Jinzhui' pistils after $24 \mathrm{~h}$ post-pollination, the expression level of Pbr036330.1 was up regulated and more than that in 'Jinzhui $x$ Yali', while Pbr010506.1 expression was down regulated. However, the differential expression of the two HRGPs reduced in pistils after $48 \mathrm{~h}$ post-pollination and there was no difference at $72 \mathrm{~h}$ post-pollination. The qRT-PCR results were consistent with the RPKMs tendency in transcriptome data of pollinated pistils (Additional file 7: Fig. S6A, B). Considering that at $24 \mathrm{~h}$ post-pollination, the self-compatible and incompatible reactions in pear pistils had already occurred [43], and that these reactions would cease at $48 \mathrm{~h}$. The data suggested that this differential expression pattern may associate with mutations of mechanisms downstream of the pollination self-compatibility reactions.

Duplicated genes within a gene pair exhibiting high homology tended to display a similar expression pattern, while our results indicated that relatively lower homology was found between HRGP gene pairs following the expansion of the superfamily. To better elucidate the characteristics of HRGP expression divergence, we examined random HRGP gene pairs versus total gene pairs (as controls). Expression divergence of HRGP genes randomly combined as gene pairs indicated the universal expression divergence among HRGPs and total gene pairs acquired from the Plant Genome Duplication Database (PGDD) indicated the universal expression divergence between duplicated genes. We found that random gene pairs, duplicated gene pairs and duplicated HRGP gene pairs presented distinct expression divergence features (Fig. 8). Expression divergence of total duplicated gene pairs likely reflected common rate of expression 


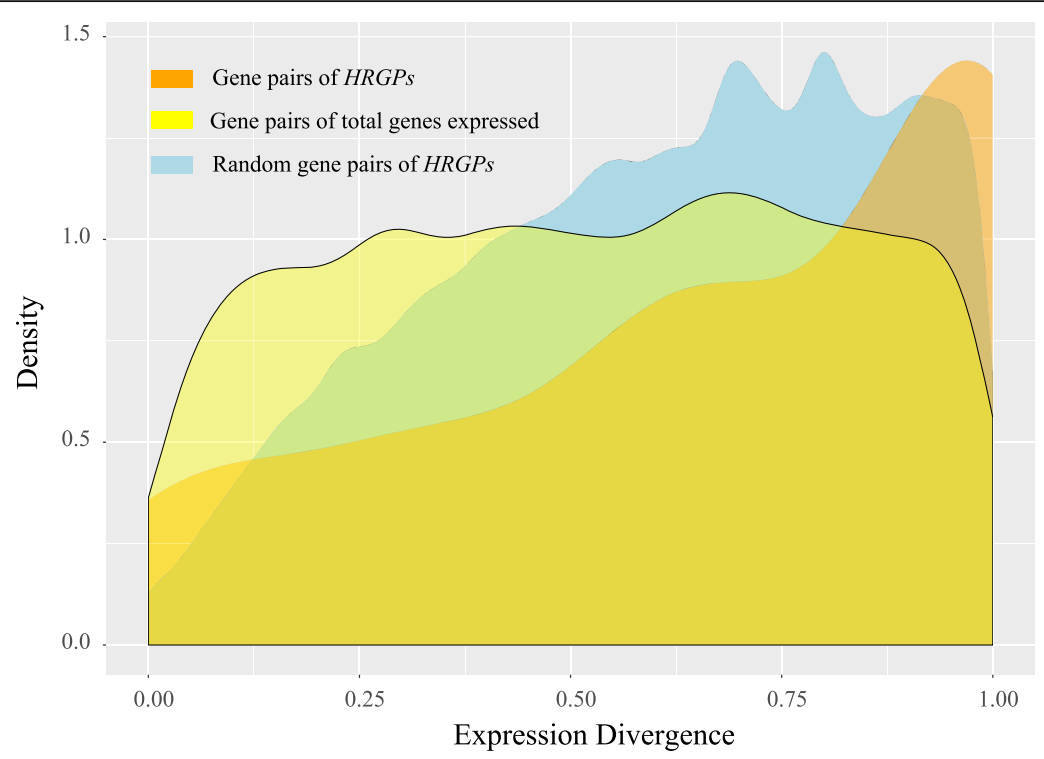

Fig. 8 Comparison of the expression divergence among HRGP gene pairs, randomly paired HRGP genes and total gene pairs. The gene pairs of totally expressed genes were acquired by MCScanX. The expression profile employed in this analysis was the RPKM value of pollinated pistils

divergence, while expression divergence of random HRGP gene pairs was represented when there was no collinear relationship. Our results indicated that the expression of HRGPs without collinear relationships was more divergent than genes from duplicated pairs. However, expression analysis of HRGP gene pairs exhibited higher divergence than both random HRGP gene pairs and total expressed gene pairs. It suggested that duplication events of HRGPs may enhance their expression divergence.

To investigate the mechanism of expression divergence of HRGPs, we also analyzed the promoter sequences of HRGP genes from WGD gene pairs. For HRGPs from WGD showed a high level of expression divergence, we compared the promoter sequences of HRGPs gene pairs from WGD. we extracted the upstream 1000 bp of HRGPs genes and predicted transcription factor bonding sites/cis elements present in the $1000 \mathrm{bp}$ upstream region. The results suggested that HRGPs gene pairs with higher expression correlation shared a couple of transcription factors while HRGPs gene pairs with higher expression divergence had no common transcription factor (Additional file 1: Table S2). It is inferred that the variation of promoter sequences in HRGPs may be an important factor accounting for expression divergence of HRGPs gene pairs from WGD events.

\section{Discussion}

A more sensitive approach of identifying HRGPs

Our examination of the expansion, evolution and expression of the HRGP gene superfamily in pear reproductive tissues highlighted key differences from those of other gene families. We employed a biased aa composition analysis for sequence collection of the HRGP superfamily instead of Blastp and Hidden Markov Model (HMM) typically used for other gene families. We employed a family-specific strategy for each family within the HRGP superfamily. The Hyp-rich motifs of each HRGP were also annotated (data not shown). Through this process, 838 genes were identified, far more than the number identified by other previously reported strategies. Indeed, in P. trichocarpa, 271 HRGPs were identified, while in Arabidopsis 162 were identified $[14,33]$. To compare our searching method with that previously reported, we searched the HRGPS in Arabidopsis and blackcotton wood and more HRGPS were acquired with our method (Additional file 1: Table S3). Although our search strategies were based on biased aa composition searching patterns of Showalter [14], a perl script written by us were applied to screen HRGPs and the searching strategy was modified to maximise the identification of HRGPs in pear. During the screening process, we used different percentage of PAST residues in candidate HRGPs using BlastP and found that a minimum of $10 \%$ was critical to identify HRGPs. Compared with BIO OHIO, our search method is more adaptive for identifying HRGPs.

\section{Both WGD and single-gene duplication are important for HRGPS}

WGD events were prevalent in several angiosperm genomes [44]. Ancient WGD events took place in the ancestral genome of angiosperm plants [45], and some 
plants experiencing multiple lineage-specific WGD events $[46,47]$. It suggested that WGD events was the major force of driving the expansion of gene families, and a large number of duplicate genes were retained from WGDs and segmental duplications [48]. However, single-gene duplication also occurred frequently in angiosperm plants [49]. Previous studies reported that pear and apple experienced two rounds of WGD events approximately in 140 MYA [50] and 30-45 MYA [51], with the two species diverging from each other in 5.421.5 MYA [37]. Our results indicated that these WGD events mainly contributed to the expansion of the HRGP family, and the extensive dispersed duplication found in HRGPs was also important. In addition, single-gene duplication events also accounted for a large proportion. An alternative explanation for the substantial proportion of dispersed duplicates detected in the HRGP family may be that duplicate genes derived from recent WGD events experienced chromosome rearrangement followed by single-gene duplication, resulting in a burst of dispersed gene duplication [52, 53]. It was reported that tandem duplication largely contributed to the expansion of some large gene families [54]; however, our results indicated that tandem duplication did not play a large role during the expansion of HRGPs.

\section{HRGPs are important for reproductive processes}

Because HRGPs were reported in many aspects of flowering plant reproductive processes [12, 39], we explored their expression pattern in pollen development and different pollinated pistils. Previous studies showed that TTS protein, $120 \mathrm{~K}$ and PELPIII are stylar glycoprotein HRGPs that could bind S-RNase in vitro in tobacco and also could interact with pollen tubes [12]. Two EXTs, Pbr036330.1 and Pbr010506.1 were abundantly expressed in pollinated pistils. Furthermore, both HRGPs exhibited differential response to SI. In pollens, Pbr036330.1 was expressed in low levels, while Pbr010506.1 was not expressed. However, the two genes showed high RPKM values in both self- and non-selfpollinated pistils. Meanwhile, we found that Pbr036330.1 and Pbr010506.1 exhibited higher expression in selfpollinated pistils than in non-self-pollinated pistils at $24 \mathrm{~h}$ post-pollination. However, these differences reduced at $48 \mathrm{~h}$, and were absent at $72 \mathrm{~h}$ (Additional file 7 : Fig. S6A, B). Previous research suggested that SI in pear usually occurred during $24 \mathrm{~h}$ post-pollination [55]. We speculated that the response process of these two HRGPs might be related to SI. Of note, the protein structures of both HRGPs contained a SPs and SP motifs, while no other conserved domains were found. However, TTS, $120 \mathrm{~K}$ and PEPLIII, which both contained Pollen Ole e I domains, contained only a $\mathrm{SP}_{n}$ motif. The relationship of the two HRGPs and SI requires further study.

The Pbr031110.1 belonged to EXT subfamily containing a particularly unique sequence of Pollen Ole e I domain. The relative expression levels of Pbr031110.1 in both pollen and style were lower than that in fruit, leaf, ovary and stem, suggesting that Pbr031110.1 was not involved in the reproductive process unlike most HRGPs (Additional file 7: Fig. S6C). With regard to the rest of the analyzed pear genes, the expression level of Pbr031110.1 was the highest in fruit, followed by ovary. Interestingly, through a blastp search of Pbr031110.1 against several species, including Prunus persica, Malus domestica, Fragaria vesca, Prunus mume, Vitis vinifera and Arabidopsis thalinana, no genes were found with a homology coefficient greater than $40 \%$ (data not shown). Pbr031110.1 contained a conserved Pollen Ole e I domain and numerous repetitive $\mathrm{SP}_{5}$ motifs which did not exist in other species, even in the closely related apple. HRGPs were associated with the polymerisation of lignin and stone cells, of which there was a particularly high content of lignin in pear fruit that was unique among flowering plants [56]. We speculated that Pbr031110.1 could account for this phenomenon.

\section{The variable Hyp-rich motifs of HRGPs impact plant evolution}

Our analysis suggested that WGD events promoted the expression divergence of HRGPs. Because duplicated genes typically exhibited more divergent expression patterns than single-copy genes [57], we compared the expression divergence of duplicated HRGPS with other common duplicated genes. This comparison revealed that the expression of duplicated HRGP is more divergent than common duplicated genes. Because the duplicated genes likely performed unique functions in different tissues and developmental stages [58], we speculated that the higher expression divergence of HRGPs may due to their highly variable Hyp-rich motifs. The structure integrity of Hyp-motifs could also impact the evolutionary rates of genes. The duplicated genes contained the same domains, and the ones with additional Hyp-rich motifs exhibited distinct evolutionary rates. Selection pressure analysis of the Pollen Ole e I and 7deoxyloganetin glucosyltransferase-like gene family suggested that gain and loss of Hyp-rich motifs are more frequent under positive selection.

The expression divergence of two genes was generally considered to be driven by random effects during a neutral-evolution model $[59,60]$. However, the extremely high expression divergence of HRGPs suggested that the evolution of HRGPS did not occur under this model. Furthermore, the high variability of Hyp-rich motifs correlated with similar variability in the expression 
of HRGPs particularly in duplicated HRGPs. Genes retained after duplication likely gained new functions in a genome, further driving expression divergence $[61,62]$. Therefore, a large number of HRGPS with high expression divergence likely constituted a major force in plant evolution.

\section{Conclusions}

Our data elucidated the expansion, evolution and expression patterns of HRGP superfamily in pear. Given the strong signal of positive selection and the drastic expression divergence between duplicated HRGP genes, we proposed that the HRGPs may play important roles in the evolution of novel functions and adaption of plants. The HRGP genes were highly rich in the pistil which might provide an important point of view for us to study reproductive process of pear.

\section{Methods}

\section{HRGP sequences collection}

All gene sequences of Chinese White pear (Pyrus bretschneideri) were downloaded from the pear genome project (http://peargenome.njau.edu.cn/) [37]. The biased aa composition was a basic criterion for collecting HRGPs [14]. Different subfamilies of HRGPs possessed various Hyp-rich motifs. The content and percentage of PAST were the criteria used to search for AGPs. Other subfamilies were identified based on PAST percentage as well as the presence of unique motifs. For FLAs, the H1 motif was used to perform a Hmmsearch [14]. For EXTAGPs, the $\mathrm{SP}_{\mathrm{n}}$ motif was used as an additional search criterion. For classical AGPs, a minimum of 30\% PAST content was required. For AG peptides, the percentage of PAST was at least $10 \%$, and the peptide length was less than 90 aa. For lysine-rich AGPs, [X]KK, KKK and $\mathrm{K}[\mathrm{X}] \mathrm{K}$ residue sequences were used as additional criteria [14]. For EXTs, more than $2 \mathrm{SP}_{\mathrm{n}}(n=3,4$ and 5) sequences were required. For PRPs, the PPV $[\mathrm{X}][\mathrm{KC}]$ sequence was required. Blastp was used to check sequences with a threshold around 10\% PAST. The SP annotations were performed by SignalP4.0 [63] and the GPI predictions by big-PI Predictor [64, 65].

\section{Gene duplication analysis of HRGP genes}

The collinear relationships of duplicated HRGPs in pear were acquired by MCScanX [44]. Collinear gene pairs were presented by Circos [66], and the genomic location of HRGPS was superimposed into the corresponding chromosomes, while unanchored HRGPS were not included in the collinearity analysis. To estimate the GC content of pear genome, we split each chromosome into $10,00 \mathrm{~kb}$ per window and calculated the GC content of each $10 \mathrm{~kb}$ window by a Perl script.
Estimation of selective pressure and evolutionary rate To compare the evolutionary rate between chimeric HRGP genes and the genes with corresponding conserved domains, KaKs_Calculator2.0 [67] was selected to calculate the pairwise $\mathrm{Ka} / \mathrm{Ks}$ values by the NG method [68]. We collected the LRRs (PF12799.5), RNA recognition motif (PF00076.20), LTP (PF14368.4), protein tyrosine kinase (PF07714.15) and pollen proteins Ole e I like (PF01190.15) by a Hmmsearch against the pear genome, with the corresponding HMM files downloaded from the pfam database (http://pfam.janelia.org/) [69]. The program Codeml in PAML was used to estimate the selection pressure [70]. Sequence alignments were performed by Muscle and displayed by JalView with default parameters [71, 72]. Hyp-motifs of PRPs were drawn by Weblogo (http://weblogo.berkeley.edu/logo.cgi) [73]. Guide trees were constructed by RAxML using CDS with GTRCATI mode [74]. In codeml, Site model was used to evaluate positively selected sites. Models M0, M7 and M8 were selected to evaluate the probability of omega. The likelihood ratio test $(\mathrm{LRT})=\operatorname{chi} 2(2 \mathrm{dl})=$ chi(abs(2*(Lnl7-Lnl8))). The Ks values were used to estimate the divergence time of Pollen Ole e I gene family. Because the Ks values of 7-deoxyloganetin glucosyltransferase-like gene pairs were insufficient to estimate the divergence time of the entire family, Ks values of the gene blocks in which the 7-deoxyloganetin glucosyltransferase-like genes located were employed. The Ks for evaluating the relative divergence time of each family was acquired from PGDD (http://chibba.agtec. uga.edu/duplication/) [75]. Divergence time, $\mathrm{T}=\mathrm{Ks} /$ $2 \lambda$, with $\lambda=9.1^{*} 10^{-9}$, which was derived from black cottonwood [76].

\section{Phylogenetic analysis}

The phylogenetic trees in our analysis were constructed using RAxML. Sequence alignments were performed by multiple sequence comparison by log-expectation (Muscle) with default parameters. Trees made with codeml were constructed by coding sequences. Other trees were constructed by amino acid sequences. For trees constructed by amino acids, model PROTGAMMA was selected and for trees constructed by nucleotides, model ASC_BINGAMMA was selected. The presentation of trees was done by the Interactive Tree of Life (http://itol.embl.de) [77] and EvolView (http://www.evolgenius.info/evolview) [78].

\section{Expression divergence analysis}

Six expression profiles of pollinated pistils of 'Jinzhui $x$ Yali' and 'Jinzhui $\mathrm{x}$ Jinzhui' were used for expression divergence analysis. The divergences in the expression of three types of gene pairs were calculated separately: (1) When gene $\mathrm{a}$ and gene $\mathrm{b}$ were in one gene pair, their expression 
RPKM in six libraries was set as two matrices, $A$ and $B$, where $\mathrm{A}=\left[\mathrm{a}_{\mathrm{k}}\right](\mathrm{k}=1 \sim 6)$ and $\mathrm{B}=\left[\mathrm{b}_{\mathrm{k}}\right](\mathrm{k}=1 \sim 6)$. (2) $\mathrm{Ex}$ pression conservation $(\mathrm{EC})$ was calculated by $\mathrm{EC}(\mathrm{i})=$ PCC (A, B). (3) The expression divergence of gene pair ' $i$ ' was computed as 1-EC (i). All calculations were performed by $\mathrm{R}$.

\section{RPKM values from RNA-seq of HRGPs in pollen and polli- nated pistils}

The RPKM values of HRGPs in pollen developmental stages and fruit developmental stage were acquired from our previous reports $[79,80]$. The pollens of 'Jinzhui' and 'Dangshansuli' were collected from Jiangpu farm of Nanjing Agricultural University. To calculate the RPKM values of HRGPs in pollinated pistils of 'Jinzhui $x$ Yali' and 'Jinzhui $x$ Jinzhui', the pistils of 'Jinzhui' and 'Yali' were all pollinated with 'Jinzhui' pollen and were collected in $24 \mathrm{~h}, 48 \mathrm{~h}$ and $72 \mathrm{~h}$ post-pollination in Jiangpu farm. All RPKM values were normalized into -3 to 3 and displayed as heat maps by $\mathrm{R}$. The expression pattern of HRGPs during the dynamic pear fruits development were acquired from NCBI and the RPKM values were normalized into -4.55 to 4.55 and displayed as heat maps by $\mathrm{R}$.

\section{qRT-PCR analysis}

Total RNA used for qRT-PCR analysis was extracted from six different stages of pistil pollination (i.e. JY24, JY48, JY72, JJ24, JJ48 and JJ72). Total RNA was adjusted to the same concentration for first-strand cDNA synthesis using TransScript One-Step gDNA Removal and cDNA Synthesis SuperMix (TransGen Biotech Co. Ltd.) according to the manufacturer's instructions. qRT-PCR analysis was carried out using LightCycler 480 SYBR GREEN I Master mix (Roche) according to the manufacturer's protocol. We performed each reaction using a $20 \mu \mathrm{l}$ mixture containing $12 \mu \mathrm{l}$ of LightCycler 480 SYBR GREEN I Master, $5 \mu \mathrm{l}$ of nuclease-free water, $1 \mu \mathrm{l}$ of each primer and $1 \mu \mathrm{l}$ of diluted cDNA. The qRT-PCR began with $5 \mathrm{~min}$ at $94^{\circ} \mathrm{C}$, followed by 55 cycles at $94^{\circ}$ $\mathrm{C}$ for $3 \mathrm{~s}, 60^{\circ} \mathrm{C}$ for $10 \mathrm{~s}$ and $30 \mathrm{~s}$ of extension at $72{ }^{\circ} \mathrm{C}$. Pyrus Tubulin (accession no. AB239681) was used as the internal control gene and the relative expression levels were calculated with the $2^{-\Delta \Delta \mathrm{Ct}}$ method. All primers were shown in Additional file 1: Table S4. RPKMs of Pbr036330.1 and Pbr010506.1 in pollinated pistils were standardized to their corresponding relative expressions in pollinated pistils.

\section{Identification of cis-regulated elements in HRGPs}

To identify the potential transcription factor in the promoter sequences of HRGPs, TSSPlant in Softberry Website (http://www.softberry.com/) was used for this analysis [81]. We extracted the 1000 bp upstream sequences of HRGPs from WGD events and screened against the TSSPlant database.

\section{Additional files}

\begin{abstract}
Additional file 1: Table S1. Genomic annotations and classifications of HRGPs in pear. SP refers to signal peptide; GPI refers to

glycosylphosphatidylinositol. Table S2. The transcription factor shared by HRGP gene pairs from WGD event. Table S3. The comparison of searching strategy between our method and the method previously reported. Table S4. Primers for HRGPS in qRT-PCR. (XLSX 114 kb)

Additional file 2: Figure. S1. Protein sequences of representative HRGPS in pear. Coloured sequences indicate the predicted signal peptides (pink) in the N-terminus, GPI anchors (light green) in the Cterminus, PA, AP, SP and TP repeats (yellow), lysine-rich regions (light blue), $\mathrm{H} 1$ tags (blue), SP $\mathrm{n}$ motifs (dark blue) and PPV[X][KC] motifs (light pink). (PDF $4458 \mathrm{~kb}$ )
\end{abstract}

Additional file 3: Figure. S2. Phylogenetic tree of the EXT family. The phylogenetic tree was constructed by RAxML using amino acid sequences. Taxa with stars indicate SP3-EXT, with gcircles indicate SP4EXT and with triangles indicate SP5-EXT. (PDF $2349 \mathrm{~kb}$ )

Additional file 4: Figure. S3. Phylogenetic tree of the PRP family. The phylogenetic tree was constructed by RAxML using amino acid sequences. The red taxa indicate PRPs with PPV $[X] C$ motifs and the blue taxa indicate PRPs with PPV[X]K motifs. (PDF 2910 kb)

Additional file 5: Figure. S4. Heatmap of the expression levels of HRGP genes in reproductive tissues. A. The expression levels of HRGP genes in pollen. MP, HP, PT and SPT correspond to four different developmental stages: matured pollen, hydrated pollen, growing pollen tubes after three hours of hydration and stopped growing pollen tubes, respectively. B. The expression levels of HRGP genes in the pistils of 'Jinzhui' pollinated with self- and non-self-pollen; JY24, JJ24, JY48, JJ48, JY72 and JJ72 refer to pollinated pistils corresponding to time after pollination. The colour scale represents $\log 2$ transformed reads per kilobase per million (RPKM) values. Light green indicates low expression and red indicates high expression. (PDF $313 \mathrm{~kb}$ )

Additional file 6: Figure. S5. Heatmap of the expression levels of HRGP in 6 stages of pear fruit. S1 S6 correspond to the dynamic stages of fruit development at $15 \mathrm{~d}, 36 \mathrm{~d}, 80 \mathrm{~d}, 110 \mathrm{~d}, 145 \mathrm{~d}$ and $167 \mathrm{~d}$ after flowering. (PDF $388 \mathrm{~kb}$ )

Additional file 7: Figure. S6. Relative expression levels of HRGPS in pollinated pistils. A-C. The relative expression levels of Pbr036330.1 (A), Pbr010506.1 (B) and Pbr031110.1 (C) in different pear tissues. JY24, JJ24, $J Y 48, J J 48, J Y 72$ and $J J 72$ refer to pollinated pistils corresponding to time after pollination with 'Yali' and 'Jinzhui' pollen. (PDF 183 kb)

\section{Abbreviations}

120 K: 120 kDa glycoprotein; A: Alanine; AGP: Arabinogalactan-protein; ECM: Extracellular matrix; EXT: Extensin; HMM: Hidden markov model; HRGP: Hydroxyproline-rich glycoprotein; Ka: Nonsynonymous substitutions per site; Ks: Synonymous substitutions per site; Muscle: Multiple sequence comparison by log-expectation; P: Proline; PCC: Pearson correlation coefficient; PELPIII: Class III pistil-specific extensin-like; RPKM: Reads per kilobase per million; SI: Self-incompatibility; T: Threonine; TTS: Transmitting tissue-specific; WGD: Whole genome duplication

\section{Acknowledgements}

We would like to express our gratitude to enago for their great assistance in our scientific language editing. We would also like to thank anonymous reviewers for comments on this manuscript.

\section{Funding}

This work was supported by the National Natural Science Foundation of China (NO.31522048, NO.31471839, and NO.31772276), the National Key Technology R\&D Program of the Ministry of Science and Technology of China (2014BAD16B03-4), and the Fundamental Research Funds for the Central Universities (KYTZ201602, KYLH201502-2). 


\section{Availability of data and materials}

The datasets generated and/or analysed in this study are available from the corresponding author on reasonable request.

Stop-growth pollen tube, Accession: SRX1356346; Pollen tube, Accession: SRX1356343; Hydrated pollen grains, Accession: SRX1356152; Mature pollen grains of pear, Accession: SRX1356151.

GSM825779: Pear fruit developmental stage 1. Accession: SRX104374; GSM825780: Pear fruit developmental stage 2. Accession: SRX104375;GSM825781: Pear fruit developmental stage 3. Accession: SRX104376; GSM825782: Pear fruit developmental stage 4. Accession: SRX104377;GSM825783: Pear fruit developmental stage 5. Accession: SRX104378.

\section{Authors' contributions}

$\mathrm{HJ}$ contributed to data analysis, preparation of figures and manuscript drafting. XL improved the methodology of data collection and analysis. SS and $C T$ contributed to collecting samples and performing the GRT-PCR experiment. $X \mathrm{Q}$, JL and $\mathrm{PW}$ contributed to analysis of HRGP evolution. JW contributed to analyzing and clarifying of data. SZ contributed to literature research and guiding the integrity of the entire study. ST conceived and designed the details of the whole experiment and revised the manuscript. he provided us the transcriptome data of the pollinated pistils and discussed with us the relevant data and participated in the language revision of the whole manuscript. All authors have read and approved the final manuscript.

\section{Ethics approval and consent to participate}

The genome database of Chinese White Pear is acquired from Center of Pear Engineering Technology Research; the pollen of 'Dangshansuli' and 'Jinzhui' and 'Yali', the styles of 'Jinzhui' and 'Yali' were all collected from Jiangpu farm in Nanjing Agricultural University. The genome database, pollen and styles are applied for our research under the permission of Center of Pear Engineering Technology Research.

\section{Consent for publication}

Not applicable.

\section{Competing interests}

The authors declare that they have no competing interests.

\section{Publisher's Note}

Springer Nature remains neutral with regard to jurisdictional claims in published maps and institutional affiliations.

Received: 25 August 2017 Accepted: 5 February 2018

Published online: 17 February 2018

\section{References}

1. Josè-Estanyol M, Puigdomènech P. Plant cell wall glycoproteins and their genes. Plant Physiol Biochem. 2000;38(1):97-108.

2. Seifert GJ, Roberts K. The biology of arabinogalactan proteins. Annu Rev Plant Biol. 2007:58:137-61.

3. Showalter AM. Introduction: plant cell wall proteins. Cell Mol Life Sci. 2001; 58(10):1361-2

4. Nothnagel EA. Proteoglycans and related components in plant cells. Int Rev Cyto. 1997;174:195-291.

5. Showalter AM. Arabinogalactan-proteins: structure, expression and function. Cell Mol Life Sci. 2001;58(10):1399-417.

6 Schindler T, Bergfeld R, Schopfer P. Arabinogalactan proteins in maize coleoptiles: developmental relationship to cell death during xylem differentiation but not to extension growth. Plant J. 1995;7(1):25-36.

7 Hijazi M, Roujol D, Nguyen-Kim H, Del Rocio Cisneros Castillo L, Saland E, Jamet E, Albenne C. Arabinogalactan protein 31 (AGP31), a putative network-forming protein in Arabidopsis thaliana cell walls? Ann. Bot. 2014; 114(6):1087-97.

8 Yang J, Sardar HS, McGovern KR, Zhang Y, Showalter AM. A lysine-rich arabinogalactan protein in Arabidopsis is essential for plant growth and development, including cell division and expansion. Plant J. 2007;49(4):629-40.

9 Cheung AY, Wang H, Wu HM. A floral transmitting tissue-specific glycoprotein attracts pollen tubes and stimulates their growth. Cell. 1995; 82(3):383-93.
10 Chen W, Stoddard FL, Baldwin TC. Developmental regulation of mannan,arabinogalactan-protein, and pectic epitopes in pistils of Vicia faba (Faba bean). Int J Plant Sci. 2006;167:919-32.

11 Du H, Simpson RJ, Clarke AE, Bacic A. Molecular characterization of a stigma-specific gene encoding an arabinogalactan-protein (AGP) from Nicotiana alata. Plant J. 1996;9(3):313-23.

12 Cruz-Garcia F, Nathan Hancock C, Kim D, McClure B. Stylar glycoproteins bind to S-RNase in vitro. Plant J. 2005;42(3):295-304.

13 Jauh GY, Eckard KV, Nothnagel EA, Lord EM. Adhesion of lily pollen tubes on an artificial matrix. Sex Plant Reprod. 1997;10(3):173-80.

14 Showalter AM, Keppler B, Lichtenberg J, Gu D, Welch LR. A bioinformatics approach to the identification, classification, and analysis of hydroxyprolinerich glycoproteins. Plant Physiol. 2010;153(2):485-513.

15 Wu H, De Graaf B, Mariani C, Cheung AY. Hydroxyproline-rich glycoproteins in plant reproductive tissues: structure, functions and regulation. CellMollife Sci. 2001;58(10):1418-29.

16 Josè M, Puigdomènech P. Structure and expression of genes coding for structural proteins of the plant cell wall. New Phytol. 1993;125(2):259-82.

17 Keller B, Lamb CJ. Specific expression of a novel cell wall hydroxyproline-rich glycoprotein gene in lateral root initiation. Genes. Dev. 1989;3(10):1639-46.

18 Ito M, Kodama H, Komamine A, Watanabe A. Expression of extensin genes is dependent on the stage of the cell cycle and cell proliferation in suspensioncultured Catharanthus Roseus cells. Plant Mol Biol. 1998;36(3):343-51.

19 Sadava D, Chrispeels MJ. Hydroxyproline-rich cell wall protein (extensin): role in the cessation of elongation in excised pea epicotyls. Dev Biol. 1973; 30(1):49-55

20 Stratford S, Barnes W, Hohorst DL, Sagert JG, Cotter R, Golubiewski A, Showalter AM, McCormick S, Bedinger P. A leucine-rich repeat region is conserved in pollen extensin-like (Pex) proteins in monocots and dicots. Plant Mol Biol. 2001;46(1):43-56.

21 Rubinstein AL, Broadwater AH, Lowrey KB, Bedinger P. Pex1, a pollen-specific gene with an extensin-like domain. ProNatlAcadSciUSA. 1995;92(8):3086-90.

22 Ringli $C$. The role of extracellular LRR-extensin (LRX) proteins in cell wall formation. Plant Biosyst. 2005;139(1):32-5.

23 Sommer-Knudsen J, Bacic A, Clarke AE. Hydroxyproline-rich plant glycoproteins. Phytochemistry. 1998;47(4):483-97.

24 Van de Wiel C, Scheres B, Franssen H, Van Lierop M-J, Van Lammeren A, Van Kammen $A$, et al. The early nodulin transcript ENOD2 is located in the nodule parenchyma (inner cortex) of pea and soybean root nodules. EMBO J. 1990;9(1):1.

25 Jose-Estanyol M, Ruiz-Avila L, Puigdomènech P. A maize embryo-specific gene encodes a proline-rich and hydrophobic protein. Plant Cell. 1992;4(4):413-23.

26 De Nettancourt D. Incompatibility and incongruity in wild and cultivated plants, vol. 3. Berlin: Springer Science \& Business Media; 2001.

27 Takayama S, Isogai A. Self-incompatibility in plants. Annu Rev Plant Biol. 2005;56:467-89.

28 Hancock CN, Kent L, McClure BA. The stylar $120 \mathrm{kDa}$ glycoprotein is required for S-specific pollen rejection in Nicotiana. Plant J. 2005;43(5):716-23.

29 Lind JL, Bönig I, Clarke AE, Anderson MA. A style-specific 120-kDa glycoprotein enters pollen tubes of Nicotiana alata in vivo. Sex Plant Reprod. 1996;9(2):75-86.

30 De Graaf BHJ, Knuiman BA, Van Der Weerden GM, Feron R, Derksen J, Mariani C. The PELPIII glycoproteins in Solanaceae: stylar expression and transfer into pollen tube walls. Sex Plant Reprod. 2004:16(5):245-52.

31 Lee $C B$, Kim S, McClure B. A pollen protein, NaPCCP, that binds pistil arabinogalactan proteins also binds phosphatidylinositol 3-phosphate and associates with the pollen tube endomembrane system. Plant Physiol. 2009; 149(2):791-802.

32 Lichtenberg J, Keppler BD, Conley T, Gu D, Burns P, Welch LR, Showalter AM. Prot-class: a bioinformatics tool for protein classification based on amino acid signatures. Nat Sci. 2012;04(12):1161-4.

33 Showalter AM, Keppler BD, Liu X, Lichtenberg J, Welch LR. Bioinformatic identification and analysis of Hydroxyproline-rich glycoproteins in Populus trichocarpa. BMC Plant Biol. 2016;16(1):229.

34 Maher C, Stein L, Ware D. Evolution of Arabidopsis microRNA families through duplication events. Genome Res. 2006;16(4):510-9.

35 Jiang W, Liu Y, Xia E, Gao L. Prevalent role of gene features in determining evolutionary fates of whole-genome duplication duplicated genes in flowering plants. Plant Physiol. 2013;161(4):1844-61.

36 Galtier N, Piganeau G, Mouchiroud D, Duret L. GC-content evolution in mammalian genomes: the biased gene conversion hypothesis. Genetics. 2001;159(2):907-11. 
37 Wu J, Wang Z, Shi Z, Zhang S, Ming R, Zhu S, Khan MA, Tao S, Korban SS, Wang $\mathrm{H}$, et al. The genome of the pear (Pyrus bretschneideri Rehd.). Genome Res. 2013;23(2):396-408.

38 Lee $\mathrm{JH}$, Waffenschmidt S, Small L, Goodenough U. Between-species analysis of short-repeat modules in cell wall and sex-related hydroxyproline-rich glycoproteins of Chlamydomonas. Plant Physiol. 2007;144(4):1813-26.

39 Clarke A, Gleeson P, Harrison S, Knox RB. Pollen-stigma interactions: identification and characterization of surface components with recognition potential. Pro.Natl.Acad.Sci.USA. 1979;76(7):3358-62

40 Wu HM, Wong E, Ogdahl J, Cheung AY. A pollen tube growth-promoting arabinogalactan protein from Nicotiana alata is similar to the tobacco TTS protein. Plant J. 2000;22(2):165-76.

41 Wu J, Li M, Li T. Genetic features of the spontaneous self-compatible mutant 'Jin Zhui'(Pyrus bretschneideri Rehd.). PLoS One. 2013;8(10):e76509.

42 Li MF, Li XF, Han ZH, Shu HR, Li TZ. Molecular analysis of two Chinese pear (Pyrus bretschneideri Rehd.) spontaneous self-compatible mutants, Yan Zhuang and Jin Zhui. Plant Biol. 2009;11(5):774-83.

43 Kaufmane E, Rumpunen K. Pollination, pollen tube growth and fertilization in Chaenomeles japonica (Japanese quince). Sci Hort. 2002;94(3):257-71.

44 Wang Y, Tang H, DeBarry JD, Tan X, Li J, Wang X, Lee T-h, Jin H, Marler B, Guo H. MCScanX: a toolkit for detection and evolutionary analysis of gene synteny and collinearity. Nucleic Acids Res. 2012;40(7):e49.

45 Bowers JE, Chapman BA, Rong J, Paterson AH. Unravelling angiosperm genome evolution by phylogenetic analysis of chromosomal duplication events. Nature. 2003;422(6930):433-8.

46 Paterson AH, Bowers JE, Chapman BA. Ancient polyploidization predating divergence of the cereals, and its consequences for comparative genomics. Pro.Natl.Acad.Sci.USA. 2004;101(26):9903-8.

47 Tang $H$, Bowers JE, Wang $X$, Ming R, Alam M, Paterson AH. Synteny and collinearity in plant genomes. Science. 2008;320(5875):486-8.

48 Wang $Y$, Wang $X$, Paterson AH. Genome and gene duplications and gene expression divergence: a view from plants. Ann N Y Acad Sci. 2012;1256(1):1-14.

49 Paterson AH, Chapman BA, Kissinger JC, Bowers JE, Feltus FA, Estill JC. Many gene and domain families have convergent fates following independent whole-genome duplication events in Arabidopsis, Oryza, Saccharomyces and Tetraodon. Trends Genet. 2006:22(11):597-602.

50 Fawcett JA, Maere S, Van de Peer Y. Plants with double genomes might have had a better chance to survive the cretaceous-tertiary extinction event. Pro.Natl.Acad.Sci.USA. 2009;106(14):5737-42.

51 Velasco R, Zharkikh A, Affourtit J, Dhingra A, Cestaro A, Kalyanaraman A, Fontana P, Bhatnagar SK, Troggio M, Pruss D. The genome of the domesticated apple (Malus X domestica Borkh.). Nat Genet. 2010;42(10):833-9.

52 Lynch M, Conery JS. The evolutionary fate and consequences of duplicate genes. Science. 2000;290(5494):1151-5.

53 Flagel LE, Wendel JF. Gene duplication and evolutionary novelty in plants. New Phytol. 2009;183(3):557-64.

54 Cannon SB, Mitra A, Baumgarten A, Young ND, May G. The roles of segmental and tandem gene duplication in the evolution of large gene families in Arabidopsis thaliana. BMC Plant Biol. 2004:4(1):1.

55 Zhou JG, Hu HL, Zhang HR, Wang J. Fluoroscope observation on selfincompatibility degree of apple and pear. Journal of Henan Institute of Science and Technology (Natural Science Edition). 2010;2:009.

56 Liyama K, Lam TBT, Meikle PJ, Ng K, Rhodes DI, Stone BA. Cell wall biosynthesis and its regulation. Forage cell wall structure and Digestibility. 1993;24:621-83.

57 Li WH, Yang J, Gu X. Expression divergence between duplicate genes. Trends Genet. 2005;21(11):602-7.

58 Markert CL. Cellular differentiation-an expression of differential gene function. In Congenital malformations. 1964;2:163.

59 Gu X. Statistical framework for phylogenomic analysis of gene family expression profiles. Genetics. 2004;167(1):531-42

60 Oakley TH, Gu Z, Abouheif E, Patel NH, Li WH. Comparative methods for the analysis of gene-expression evolution: an example using yeast functional genomic data. Mol BiolEvol. 2005;22(1):40-50.

61 Force A, Lynch M, Pickett FB, Amores A, Yan Y-L, Postlethwait J. Preservation of duplicate genes by complementary, degenerative mutations. Genetics. 1999;151(4):1531-45

62 Lynch M, Force $A$. The probability of duplicate gene preservation by subfunctionalization. Genetics. 2000;154(1):459-73.

63 Petersen TN, Brunak S, von Heijne G, Nielsen H. SignalP 4.0: discriminating signal peptides from transmembrane regions. Nat Methods. 2011;8(10):785-6
64 Eisenhaber B, Bork P, Eisenhaber F. Prediction of potential GPI-modification sites in proprotein sequences. J Mol Biol. 1999:292(3):741-58.

65 Eisenhaber B, Bork P, Yuan Y, Löffler G, Eisenhaber F. Automated annotation of GPI anchor sites: case study C. Elegans. Trends. Biochem. Sci. 2000;25(7):340-1.

66 Krzywinski M, Schein J, Birol I, Connors J, Gascoyne R, Horsman D, Jones SJ, Marra MA. Circos: an information aesthetic for comparative genomics. Genome Res. 2009;19(9):1639-45.

67 Wang D, Zhang Y, Zhang Z, Zhu J, Yu J. KaKs_Calculator 2.0: a toolkit incorporating gamma-series methods and sliding window strategies. Genomics, Proteomics \& Bioinformatics. 2010;8(1):77-80.

68 Nei M, Gojobori T. Simple methods for estimating the numbers of synonymous and nonsynonymous nucleotide substitutions. Mol Biol Evol. 1986:3(5):418-26.

69 Bateman A, Coin L, Durbin R, Finn RD, Hollich V, Griffiths-Jones S, Khanna A, Marshall M, Moxon S, Sonnhammer ELL. The Pfam protein families database. Nucleic Acids Res. 2004;32(suppl 1):D138-41.

70 Yang Z. PAML 4: phylogenetic analysis by maximum likelihood. MolBiol Evol. 2007;24(8):1586-91.

71 Edgar RC. MUSCLE: multiple sequence alignment with high accuracy and high throughput. Nucleic Acids Res. 2004;32(5):1792-7.

72 Waterhouse AM, Procter JB, Martin DMA, Clamp M, Barton GJ. Jalview version 2-a multiple sequence alignment editor and analysis workbench. Bioinformatics. 2009;25(9):1189-91.

73 Crooks GE, Hon G, Chandonia J-M, Brenner SE. WebLogo: a sequence logo generator. Genome Res. 2004;14(6):1188-90

74 Stamatakis A. RAxML-VI-HPC: maximum likelihood-based phylogenetic analyses with thousands of taxa and mixed models. Bioinformatics. 2006; 22(21):2688-90

75 Lee $\mathrm{TH}$, Tang H, Wang X, Paterson AH. PGDD: a database of gene and genome duplication in plants. Nucleic Acids Res. 2013;41(D1):D1152-8.

76 Tuskan GA, Difazio S, Jansson S, Bohlmann J, Grigoriev I, Hellsten U, Putnam N, Ralph S, Rombauts S, Salamov A. The genome of black cottonwood, Populus trichocarpa (Torr. \& gray). Science. 2006:313(5793):1596-604.

77 Letunic I, Bork P. Interactive tree of life (iTOL) v3: an online tool for the display and annotation of phylogenetic and other trees. Nucleic Acids Res. 2016;44(W1):W242-5

78 He Z, Zhang H, Gao S, Lercher MJ, Chen WH, Hu S. Evolview v2: an online visualization and management tool for customized and annotated phylogenetic trees. Nucleic Acids Res. 2016:44(W1):W236-41.

79 Zhou H, Yin H, Chen J, Liu X, Gao Y, Wu J, Zhang S. Gene-expression profile of developing pollen tube of Pyrus bretschneideri. Gene Expr Patterns. 2016; 20(1):11-21

80 Zhang MY, Xue C, Xu L, Sun H, Qin M-F, Zhang S, Wu J. Distinct transcriptome profiles reveal gene expression patterns during fruit development and maturation in five main cultivated species of pear (Pyrus L.). Sci Rep. 2016:6:28130.

81 Shahmuradov IA, Umarov RK, Solovyev W. TSSPlant: a new tool for prediction of plant pol II promoters. Nucleic Acids Res. 2017:45(8):e65-5.

\section{Submit your next manuscript to BioMed Central and we will help you at every step:}

- We accept pre-submission inquiries

- Our selector tool helps you to find the most relevant journal

- We provide round the clock customer support

- Convenient online submission

- Thorough peer review

- Inclusion in PubMed and all major indexing services

- Maximum visibility for your research

Submit your manuscript at www.biomedcentral.com/submit
) Biomed Central 